\title{
Primer registro de trauma en Chile. Análisis de 2 años en un hospital público
}

\author{
Juan P. Ramos P. ${ }^{1}$, Pablo R. Ottolino L. ${ }^{1}$, Carolina A. Muñoz A. ${ }^{1}$, \\ José E. Ruiz C. ${ }^{1}$, Claudia E. Arenas P. ${ }^{1}$, Francisca P. Salazar M. ${ }^{1}$, \\ Lázaro Calderón R. ${ }^{1}$, Pablo Achurra T. ${ }^{2}$ y Alfonso Díaz F. ${ }^{1}$
}

\section{First trauma registry in Chile. 2 years analysis in a public hospital}

Introduction: Trauma represents a real challenge for health systems because is a major public health problem. In Chile, the management of polytrauma patients is unknown. Aim: Describe the results of the first online trauma registry; in patients treated at the Dr. Sótero del Río Hospital during the first two years of implementation. Materials and Method: Prospective information is collected in a network registry, about trauma victims, admitted to Dr. Sótero del Río Hospital for two years. These variables involve the entire clinical care process from admission to discharge or death. Results: 3.515 admissions of trauma victims are registere in two years. Among these, $81.3 \%$ are men. $27 \%$ suffered penetrating trauma and $59 \%$ closed. They emphasize larger injuries by firearm than by bladed weapon. $18.4 \%$ of admitted patients have ISS greater than 15 points. 34 patients enter cardio-respiratory arrest and $7.3 \%$ make it hypotensive. $1,856 \mathrm{CT}$ scans are performed and $54.4 \%$ require emergency surgery. They admitted 692 thoracic trauma, 654 abdominal, $1550 \mathrm{limb}, 687$ skull injuries and 190 cervical trauma. The massive transfusion protocol is activated at $3.1 \%$ of the admissions. $8.3 \%$ use the critical patient unit and mortality is $2.9 \%$, with cranial brain trauma as the first cause and hemorrhagic shock as a second cause. Conclusions: Our hospital has a high incidence of trauma. The RT implementation allows us to know the dimension and assess the care process about the trauma patient. It is necessary to direct resources and implement training in high-volume trauma centers, as well as continuing with the extension of the tool as a multi-center monitoring strategy. Key words: registry, trauma; epidemiology; politraumatized.

\section{Resumen}

Introducción: El trauma representa un verdadero reto para los sistemas sanitarios por ser un gran problema de salud pública. En Chile se desconoce el manejo del paciente politraumatizado. Objetivo: Describir los resultados del primer registro de trauma (RT) en línea, en los pacientes que se atienden en el Hospital Dr. Sótero del Río durante los primeros dos años de la implementación. Materiales y Método: Se recoge información prospectiva en un registro en red, sobre pacientes víctimas de trauma, ingresados al Hospital Dr. Sótero del Río durante dos años. Estas variables involucran todo el proceso de atención clínica desde el ingreso al alta o fallecimiento. Resultados: En dos años, se registran 3.515 ingresos de pacientes víctima de trauma. Entre estos, el 81,3\% son hombres. El 27\% sufre trauma penetrante y $59 \%$ cerrado. Destacan más lesiones por arma de fuego que por arma blanca. El 18,4\% de los pacientes ingresados tiene un el Injury Severity Score (ISS) mayor a 15 puntos. 34 pacientes ingresan en paro cardiorrespiratorio y 7,3\% lo hace hipotenso. Se realizan 1.856 tomografías y el 54,4\% requiere cirugía de urgencia. Ingresan 692 traumas torácicos, 654 abdominales, 1.550 de extremidades, 687 lesiones en cráneo y 190 traumas cervicales. Se activa el protocolo de transfusión masiva en el 3,1\% de los ingresos. El 8,3\% utiliza la unidad de paciente crítico y la mortalidad es de $2,9 \%$, teniendo como primera causa el trauma encéfalocraneano y segunda el shock hemorrágico. Conclusiones: Nuestro hospital tiene una gran incidencia de trauma. La implementación de un RT permite conocer la dimensión y evaluar el proceso asistencial en torno al paciente víctima de trauma. Es necesario dirigir recursos e implementar capacitación en los centros de alto volumen de trauma, además, de continuar con la extensión de la herramienta como estrategia de monitorización multicéntrica.

Palabras clave: registro; trauma; epidemiología; politraumatizado.
'Hospital Dr. Sotero del Río. Santiago, Chile. 2Pontificia Universidad Católica de Chile. Santiago, Chile.

Recibido 2019-11-05 y aceptado 2020-02-12.

Correspondencia a: Dr. Juan P. Ramos P jramos.med@gmail.com 


\section{Introducción}

El trauma está considerado como una verdadera epidemia, al ser la primera causa de muerte en menores de 45 años, causando alrededor de 1,6 millones de muertes por año ${ }^{1}$. Actualmente, esta condición representa un reto para los sistemas sanitarios por ser considerado un problema de salud pública ${ }^{2,3}$.

El paciente con trauma grave, requiere de un manejo multidisciplinario precoz, además de una organización integral del personal de salud y disponibilidad de recursos en forma rápida y eficaz. El primer paso para mejorar la atención de este tipo de pacientes es el conocimiento de la epidemiología del trauma lo que permite identificar los factores relacionados con los diferentes tipos de lesiones, el manejo pre e intrahospitalario, además de su evolución ${ }^{4}$.

Los registros de trauma nacen en los años 80 como un recurso para reunir datos y generar modelos predictivos de mortalidad basado en el Injury Severity Score (ISS) ${ }^{5}$. Posteriormente nace la iniciativa de crear el National Trauma Data Bank (NTDB) que reúne información de forma prospectiva de más de un millón de pacientes de 405 centros de trauma en EE. UU. convirtiéndose así en una potente herramienta epidemiológica, de gestión en salud, investigación y destinación de recursos ${ }^{6,7}$, además, de generar políticas de prevención ${ }^{8,9}$.

Actualmente en nuestro país no existen registros de trauma, por tanto, se desconoce su demografía exacta así como su manejo intrahospitalario y evolución posterior al egreso.

El complejo hospitalario Dr. Sótero del Río, atiende a una población de 1,5 millones de habitantes, siendo centro de referencia de traumatismo encefalocraneano, trauma raquimedular y maxilofacial para toda el área suroriente de Santiago. El hospital cuenta con la infraestructura adecuada para la atención de pacientes politraumatizados en lo que refiere a 55 camas de paciente crítico e intermedio, 14 pabellones de los cuales 2 son de la unidad de emergencia adulto con disponibilidad 24 h, 3 angiógrafos y radiología intervencional. Además, cuenta con la totalidad de especialidades médico-quirúrgicas para el manejo multidisciplinario, incluyendo cirujanos de trauma con función docente asistencial activa en la Unidad de Trauma y Urgencia (UTU).

El objetivo del presente trabajo es describir la experiencia en la implementación del primer registro de trauma en línea, en los pacientes que se atienden en el Hospital Dr. Sótero del Río en los primeros dos años de la implementación del registro.

\section{Materiales y Método}

Estudio descriptivo retrospectivo de los primeros 3.515 casos ingresados en el registro de trauma del Hospital Dr. Sótero del Río.

Nuestro registro de trauma nace como un proyecto de investigación el año 2016 y ha servido como pilar fundamental para la creación del equipo de urgencia y trauma. Consta de un software disponible en el siguiente portal www.registrodetrauma. $\mathrm{cl}$ (Figura 1) que funciona online y dispone de 85 variables, las cuales una vez completadas quedan almacenadas en un servidor virtual en forma encriptada. Esta herramienta se encuentra a cargo de una enfermera de investigación y múltiples registradores que ingresan la información en forma prospectiva y diaria desde el ingreso hasta el momento del alta, traslado o fallecimiento del paciente.

Dentro de los criterios de ingreso para el registro encontramos a todo paciente hospitalizado por trauma penetrante o contuso, trauma encefalocraneano, paciente quemado y mano gravemente lesionada.

Las variables registradas van desde el ámbito prehospitalario, datos demográficos, primera atención en área de reanimación, índices de gravedad, datos intraoperatorios, en unidad de paciente crítico y sala de hospitalización.

Actualmente se encuentran en red tres hospitales situados en el área del Servicio de Salud Metropoli-

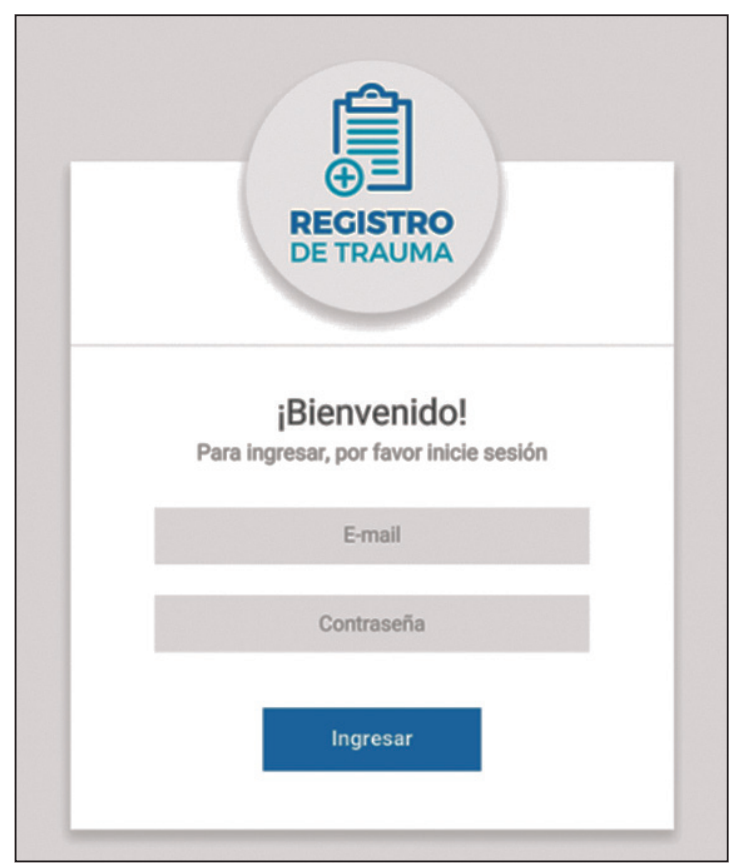

Figura 1. Plataforma electrónica e interconectada del registro de trauma. 
tano Sur Oriente (Hospital Dr. Sótero del Río, Hospital Padre Hurtado, Hospital La Florida Dra. Eloísa Díaz) y uno en Servicio de Salud Metropolitano central (Hospital de Urgencia y Asistencia Pública).

El trabajo y su desarrollo qué aceptado por el Comité de Ética del Servicio de Salud Metropolitano Sur Oriente.

Los datos analizados en este trabajo fueron exportados a una base de archivos binarios con previa autorización del Comité de Ética del Servicio de Salud Metropolitano Sur Oriente y comprende sólo a los pacientes ingresados en el Hospital Dr. Sótero del Río. Se realiza un análisis descriptivo presentando variables categóricas como proporción y las variables continuas como media, mediana, promedio, desviación estándar y rangos intercuartílicos.

\section{Resultados}

\section{Demografía}

Dentro de un periodo de 730 días (1/1/2017$31 / 12 / 18$ ) ingresan al registro 3.515 pacientes con una mediana de edad de 40,3 años (rango, 13-94 años), de los cuales el 81,3\% son hombres.

Como mecanismo de trauma destaca un $27 \%$ de trauma penetrante, $59 \%$ trauma cerrado, $10 \%$ heridas complejas y $3 \%$ pacientes quemados (Figura 2).
La mayor incidencia de heridas por arma de fuego sufridas por los pacientes que ingresan al registro ocurrió en las poblaciones Bajos de Mena, Nocedal, San Carlos, Carol Urzúa y Creta Sur. Todos pertenecientes a la comuna de Puente Alto (Figura 3). Con respecto al índice de severidad (ISS) encontramos 2.555 pacientes $(72,7 \%)$ con ISS menor a 15,646 pacientes $(18,4 \%)$ con ISS entre 15 y 24 y 305 pacientes $(8,7 \%)$ con ISS mayor a 25 .

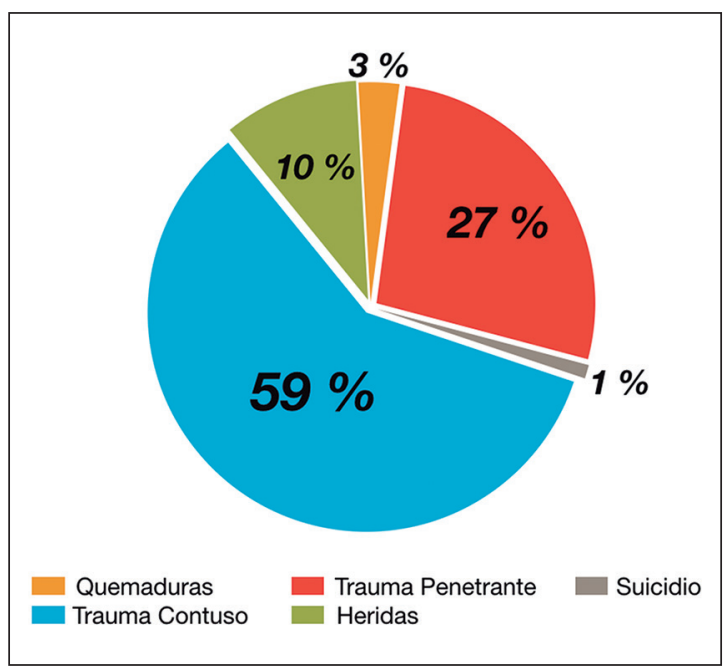

Figura 2. Distribución del mecanismo de trauma que sufrieron los pacientes ingresados.

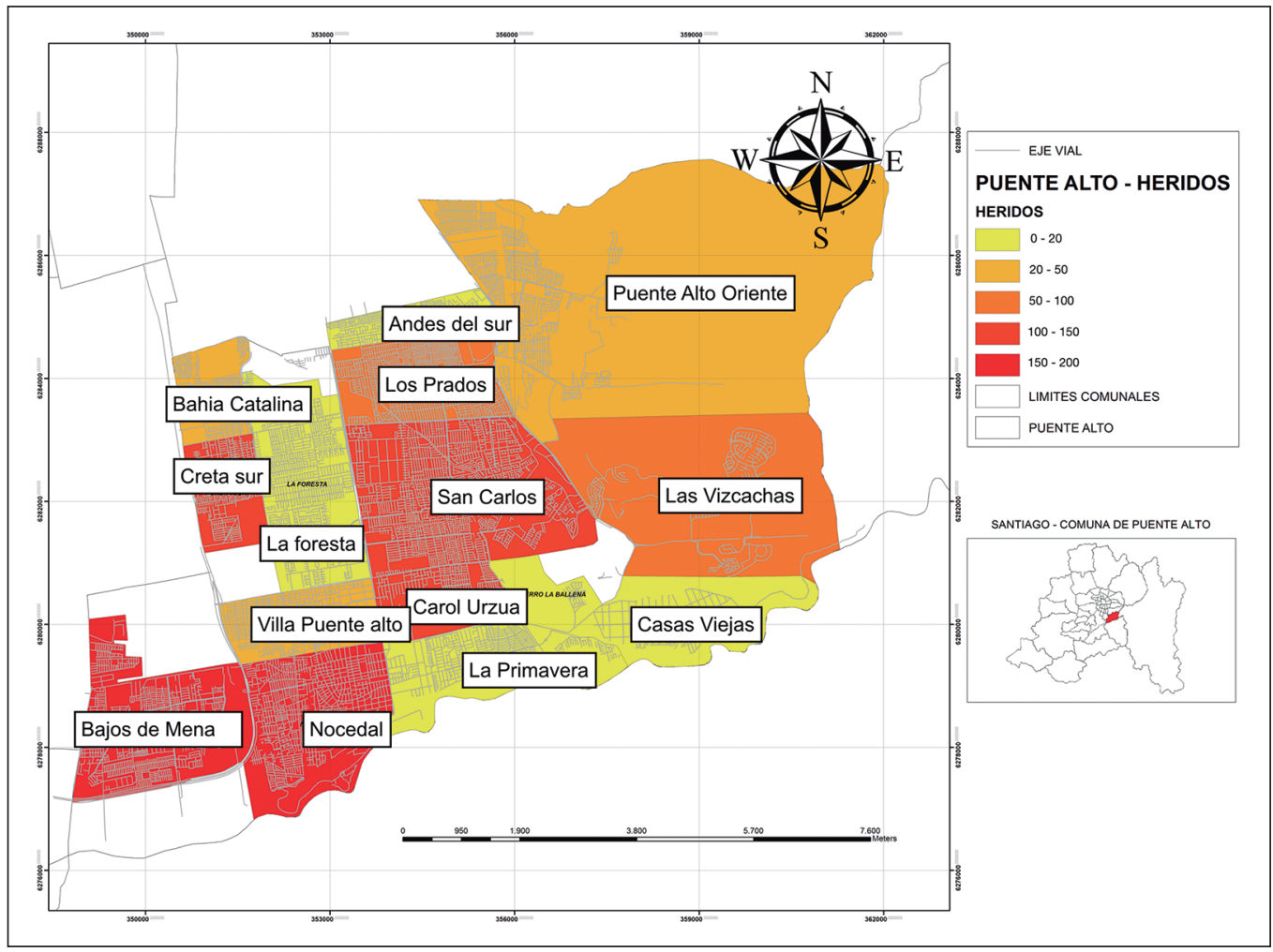

Figura 3. Distribución de la zona comunitaria en Puente Alto donde los pacientes ingresados al registro sufrieron heridas por arma de fuego. 


\section{Reanimación}

De los pacientes ingresados, $256(7,3 \%)$ lo hace hipotenso a la unidad de reanimación, $928(26,4 \%)$ taquicárdicos y $34(0,9 \%)$ en paro cardiorrespiratorio (PCR). La escala de coma de Glasgow es de 15 puntos en el $85,8 \%$, de $14-9$ puntos en el $9,4 \%$ y menor o igual a 8 puntos en el $3,8 \%$ de los pacientes. La distribución de las lesiones que presentan los pacientes registrados fueron las que muestra la Figura 4.

De los procedimientos realizados destaca que 158 pacientes $(4,5 \%)$ requieren de intubación orotraqueal en el servicio de urgencias y otros procedimientos invasivos como lo muestra la Tabla 1.

Se realiza tomografía al ingreso a 1.856 pacientes $(52,8 \%)$. El $54,4 \%$ de los ingresos requerió cirugía dentro de la hospitalización.

Se realizaron 20 toracotomías de resucitación, con una supervivencia global de 10\% (2 pacientes), al estratificarlos por mecanismo de trauma encontramos $16(80 \%)$ por trauma penetrante (TP) y $4(20 \%)$ por trauma cerrado (TC), con una supervivencia de $2(12,5 \%)$ para TP y $0(0 \%)$ para TC (Tabla 2$)$. De los dos supervivientes 1 evoluciona con secuelas neurológicas graves y otro sin secuelas.



Figura 4. Topografía corporal de las lesiones presentadas en los pacientes ingresados

\section{Cirugía}

De los 1.912 pacientes que requirieron intervención quirúrgica, la distribución según el servicio que interviene al paciente es la siguiente: traumatología $1.356(70,9 \%)$, cirugía $478(25 \%)$ y neurocirugía 105 (5,5\%) (Figura 5). El 13,5\% requerirá más de una cirugía.

El aporte de fluidos intraoperatorio en promedio fue de $1.314 \mathrm{~mL}$ (rango 500-5.000) y $9 \%$ requirieron drogas vasoactivas. Se transfundieron 267 pacientes $(7,6 \%)$ principalmente dentro de las primeras $24 \mathrm{~h}$ en base a concentrado de glóbulos rojos, plasma fresco congelado, plaquetas y crioprecipitado (Tabla 3 ). Se activa el protocolo de transfusión masiva en el 3,1\% de los pacientes. El 2,6\% de los pacientes tuvieron transfusión masiva según la definición clásica (más de 10 unidades de glóbulos en 24 h) y el 3,9\% pacientes según el criterio dinámico (igual o más de 3 unidades de glóbulos en la primera hora de ingreso).

\section{Unidad de paciente crítico}

El 8,3\% de los pacientes utiliza la unidad de paciente crítico dentro de su manejo intrahospitalario. El promedio de días en unidad de paciente crítico es de 9,8 días (1-43 días) y en unidad de paciente intermedio de 11,1 días (1-37 días). El 5,2\% de los pacientes necesitaron drogas vasoactivas (DVA), teniendo una duración promedio de 1,7 días (1-9), siendo la noradrenalina (NA) la droga utilizada en

Tabla 1. Procedimientos invasivos realizados en el box de trauma

\begin{tabular}{|lrl|}
\hline Tipo de procedimiento & n & \% \\
\hline Drenaje pleural & 283 & 8 \\
\hline Toracotomía de resucitación & 20 & 0,5 \\
\hline Denudación venosa & 43 & 1,2 \\
\hline Cricotiroidectomía & 2 & 0,05 \\
\hline Cesárea de resucitación & 2 & 0,05 \\
\hline
\end{tabular}

Tabla 2. Toracotomías de reanimación realizadas a los pacientes que ingresaron en PCR al box de trauma

\begin{tabular}{|lrcc|}
\hline & n & Supervivencia & $\%$ \\
\hline Trauma penetrante & 16 & 2 & 12,5 \\
Trauma contuso & 4 & 0 & 0 \\
Total toracotomías & 20 & 2 & 10 \\
\hline
\end{tabular}






Figura 5. Distribución de las intervenciones quirúrgicas realizadas por los equipos implicados en la atención del paciente con trauma.

todos los casos. El 7\% requirió ventilación mecánica invasiva (VMI), con una duración promedio de 7,3 días (1-30). El modo ventilatorio más usado fue volumen control, aplicándose ventilación protectora en todos los pacientes.

\section{Mortalidad y hospitalización}

La mortalidad de la serie es de $2,9 \%$ que corresponde a 102 pacientes fallecidos durante la atención. De estos el $51,4 \%$ fallece en la unidad de paciente crítico, $25,7 \%$ en la unidad de emergencia, $14,3 \%$ en pabellón y el $8,6 \%$ en la unidad de recuperación. La principal causa de muerte fue el trauma encéfalo-craneano con 51,4\% seguido por el shock hemorrágico en el $34,3 \%$. La estadía hospitalaria fue en promedio de 6,1 días con un rango entre 1-125 días. Destacan con mayor tiempo de hospitalización los pacientes neurológicos.

\section{Discusión}

Se ha logrado implementar una nueva herramienta epidemiológica en cuatro centros de referencia en salud de la región Metropolitana de Santiago de Chile. Esta compila información del paciente víctima de trauma en lo que respecta a variables demográficas, atención primaria, intraoperatorias y posterior manejo ya sea en unidad de paciente crítico o sala de hospitalización. Con los datos
Tabla 3. Hemoderivados utilizados durante las primeras $24 \mathrm{~h}$ del ingreso

\begin{tabular}{|lcc|}
\hline Hemoderivado & $\mathbf{N}^{\mathbf{0}}$ de pacientes & Unidades utilizadas \\
Glóbulos rojos & 362 & 2.238 \\
Plasma fresco congelado & 182 & 1.240 \\
Plaquetas & 102 & 853 \\
Crioprecipitado & 78 & 789 \\
\hline
\end{tabular}

presentados en este trabajo, establecemos un perfil epidemiológico que, posteriormente, dio paso a protocolizar el manejo intrahospitalario y desarrollar la Unidad de Trauma y Urgencia del Hospital Dr. Sótero del Río.

Los registros de trauma no solo proporcionan el beneficio de la información específica con respecto a la atención del paciente víctima de trauma, sino también ayudan al desarrollo de investigación clínica y son primordiales para la elaboración de un sistema de trauma con la subsecuente mejoría de la calidad de atención ${ }^{10,11}$, además del aporte en el desarrollo de políticas públicas de prevención ${ }^{12}$. Dentro de este tipo de pacientes encontramos el subgrupo de politraumatizados, actualmente cubierto por las Garantías Explícitas en Salud (GES) en nuestro país. La factibilidad de cada centro para implementar la herramienta se evalúa en base al registro electrónico de cada lugar (ficha clínica), además de la disponibilidad de un registrador. Previo comienzo de la recolección de datos, se visita cada centro y se realiza un análisis del flujo de información, además de una breve capacitación sobre la plataforma electrónica.

Este último punto (el registrador) es crítico en nuestro registro y es el principal problema al desarrollar la expansión hacia otros centros. Cada hospital, debe constar con un funcionario con dedicación exclusiva al registro, lo cual en muchos hospitales es difícil al no contar con cargos para esta función.

Dentro de la demografía de los pacientes ingresados en nuestro hospital, destaca una mayor cantidad de hombres, proporción compatible con otras series $^{13,14}$ y una edad promedio de 40,3 años, siendo esta mayor a la entregada por la literatura ${ }^{15}$.

Al determinar el mecanismo de trauma, destaca que un $27 \%$ de los pacientes sufre un trauma penetrante de los cuales característicamente encontramos mayor número de heridas por arma de fuego (517 pacientes) que por arma blanca (432 pacientes). Esto refleja un alto nivel de violencia y el aumento del uso de armas en la población de Puente Alto. 
Dentro de las variables analizadas se encuentra el lugar geográfico en donde se sufrió el trauma. Si estratificamos a los pacientes que sufrieron heridas por arma de fuego por población dentro de la comuna de Puente Alto, podemos ubicar las "zonas rojas", las cuales representarían un mayor nivel de violencia dentro del área geográfica de influencia de nuestro hospital, dentro de las cuales destacan la población Bajos de Mena, Villa Nocedal, San Carlos, Creta Sur y Carol Urzúa. El análisis de esta distribución sectorial nos lleva a plantear mejoras en el ámbito de capacitación en la atención primaria del trauma y en un sistema de rápida derivación a nuestro hospital por parte de los servicios de atención primaria de urgencia ubicados en esos sectores; además de alertar a las autoridades gubernamentales de la necesidad de políticas de seguridad y prevención más estrictas.

Dentro de los requerimientos mínimos de volumen que menciona el Colegio Americano de Cirujanos, para clasificar como centro de trauma Nivel I, se encuentra tener más de 1.200 ingresos al año por trauma o más de 240 ingresos con ISS mayor a 15 Ptos. Dentro del registro, encontramos 3.515 pacientes ingresados y 951 con ISS mayor 15 puntos en dos años, cumpliendo con ambos requisitos. Creemos que este volumen de pacientes es la realidad en múltiples hospitales que funcionan como centro de derivación siendo una necesidad dirigir recursos a estos centros e implementar protocolos que mejoren la atención de este tipo de pacientes. Este último punto es lo que justifica la creación de la Unidad de Trauma y Urgencia de nuestro centro.

De las 20 toracotomías de resucitación (reanimación) realizadas por PCR traumático, la supervivencia global de $10 \%$ algo mayor a la reportada por Asencio $^{13}$ de $7,8 \%$, de la misma manera se presentó un ligero incremento de la sobrevida en trauma penetrante de $12,5 \%$ en contraposición de $11,1 \%$ presentada por el mismo autor. Finalmente, ningún paciente sobrevivió por trauma cerrado en contraposición al $1,6 \%$ presentado por Asencio. Sin embargo, no podemos hacer conclusiones al respecto dada la necesidad de compilar un número mayor de casos.

El protocolo de transfusión masiva se activó en el $3,1 \%$ de los ingresos y se hizo efectivo en el 2,6\% si consideramos la definición clásica de transfusión masiva, porcentaje que se encuentra dentro de lo aceptable en la literatura ${ }^{15}$. Esto va de la mano con la implementación del protocolo de transfusión masiva en base a 1:1:1 y utilización de pruebas viscoelásticas en la posterior reanimación. Al 52,8\% de los ingresos se le realizó tomografía, recurso que en nuestro hospital se encuentra altamente disponible a pocos metros de la unidad de reanimación y cuenta con protocolo estricto. Su utilización se encuentra dentro de lo descrito en la literatura ${ }^{16,17}$.

La principal intervención quirúrgica es la de tipo traumatológica, seguido por la quirúrgica, que involucra extremidades, torso y cuello, esto es compatible con otros reportes de la literatura ${ }^{18}$.

El 8,3\% de los pacientes utiliza la unidad de paciente crítico, porcentaje que no refleja el número de pacientes que requirió tratamiento intensivo, debido a que este es un recurso escaso y de alta ocupabilidad dentro de nuestro hospital. Los periodos más largos tanto de utilización de cama crítica como de ventilación mecánica son debidos al paciente con neurotrauma, que llega hasta los 43 días de ocupación cama.

De los pacientes fallecidos, el 37,1\% ingresó en paro cardiorrespiratorio y el $22,9 \%$ hipotenso. La principal causa de muerte recae en el trauma encefalocraneano, esto en contexto de ser nuestro hospital centro de referencia para toda el área. La segunda causa de muerte es por shock hemorrágico $(34,3 \%)$ en donde el $86 \%$ de los pacientes tenían una lesión vascular, principalmente de grandes vasos. Otras causas de muerte son sepsis, taponamiento cardiaco, falla multiorgánica, todas con un $2,9 \%$.

Las principales limitaciones de nuestro trabajo están en la exclusión de pacientes menores de 15 años, esto actualmente, está en implementación con la creación de un registro pediátrico. Nuestro registro solo es de pacientes hospitalizados, no reflejando las consultas totales por trauma en nuestro hospital. Para este punto, es necesario realizar una revisión del dato electrónico de atención en el centro o tener un registrador $24 \mathrm{~h}$ todos los días, lo cual no es posible por su alto costo.

Como conclusión se logró implementar un sistema de registro en línea con información diaria y prospectiva de los pacientes hospitalizados víctima de trauma. Este análisis nos permite conocer los aspectos epidemiológicos de la población víctima de trauma tratada en nuestro centro. Nuestro hospital recibe un gran volumen de pacientes con trauma, característicamente con alta incidencia de trauma penetrante. Esto justifica esfuerzos gubernamentales en dirigir recursos y continuar con la capacitación y la protocolización en el manejo de este tipo de paciente. Consideramos que es necesario avanzar en la consolidación y mejora de este sistema de registro como estrategia de monitorización multicéntrica para implementar una futura regionalización en la atención del paciente con trauma. 


\section{Responsabilidades éticas}

Protección de personas y animales. Los autores declaran que para esta investigación no se han realizado experimentos en seres humanos ni en animales.
Confidencialidad de los datos. Los autores declaran que en este artículo no aparecen datos de pacientes.

Conflictos de interés: no hay.

\section{Bibliografía}

1. Matsumoto S, Jung K, Smith A, Yamazaki M, Kitano M, Coimbra R. Comparison of trauma outcomes between Japan and the USA using national trauma registries. Trauma Surgery \& Acute Care Open 2018;3:e000247. doi: 10.1136/tsaco-2018-000247.

2. Masella C, Pinho V, Costa Passos A, Spencer Netto F, Rizoli S, et al. Temporal distribution of trauma deaths: quality of trauma care in a developing country. J Trauma 2008;65:653-8. https:// doi:10.1097/TA.0b013e3181802077.

3. Pfeifer R, Tarkin I, Rocos B, Pape H. Patterns of mortality and causes of death in polytrauma patients-Has anything changed? Injury Int J Care Injured 2009;40:907-11. https://doi:10.1016/j. injury.2009.05.006.

4. Alexandrescu R, O'Brien S, Lecky F. A review of injury epidemiology in the UK and Europe: some methodological considerations in constructing rates. BMC Public Health 2009;9:226. https:// doi:10.1186/1471-2458-9-226.

5. Holbrook TL, Anderson JP, Sieber WJ, Browner D, Hoyt DB. Outcome after major trauma: 12-month and 18-month follow up results from the trauma recovery project. J Trauma 1999:46:76571

6. Chico-Fernández M, Llompart-Pou JA, Guerrero-López F, Sánchez-Casado M,
García-Sáez I, Mayor-García MD, et al. Epidemiology of severe trauma in Spain. Registry of trauma in the ICU (RETRAUCI). Pilot phase. Med Intensiva 2016;40:327-47. https://doi: 10.1016/j. medin.2015.07.011.

7. Søreide K. Epidemiology of major trauma. Br J of Surg. 2009;96:697-8. https:// doi:10.1002/bjs.6643.

8. MacKenzie E, Rivara F, Jurkovich G, Nathens A, Frey K, Egleston B, et al. A National Evaluation of the Effect of Trauma-Center Care on Mortality. N Engl J Med. 2006;354:366-78. https:// doi:10.1056/NEJMsa052049

9. Peitzman A, Puyana JC, Ivatury R. Trauma. Sociedad Panamericana de Trauma. Segunda Ed. 2009. p. 20-33.

10. Nwomeh BC, Lowell W, Kable R, Haley K, Ameh EA. History and development of trauma registry: lessons from developed to developing countries. World journal of emergency surgery: WJES 2006;1:32.

11. Marina-Martínez L, Sánchez-Casado M, Hortiguela-Martin V, Taberna-Izquierdo MA, Raigal-Caño A, Pedrosa-Guerrero A. RETRATO (REgistro deTRAuma grave de la provincia de TOledo): visión general y mortalidad. Med Intensiva 2010;34:37987.

12. Ordóñez CA, Pino LF, Tejada JW, Badiel M, Loaiza JH, Mata L V, et al. Experience of two first level hospitals in the southwest region of Colombia on the implementation of the Panamerican Trauma Society International Trauma Registry. Revista do Colégio Brasileiro de Cirurgiões 39:255-62.

13. Asencio JA, Berne JD, Demetriades D, Chang L, Murray J, Falabella A, et al. One hundred five penetrating cardiac injuries: A 2-year prospective evaluation. J Trauma 1998;44:1073-82.

14. Ruiz C, Mimica X, Lisbona M, Donoso J, Arriagada P, Roa M, et al. Características de los pacientes traumatizados que ingresan a la UCI de un hospital general en Chile. Rev Med Chile 2013;141:1389-94. https://doi:10.4067/S003498872013001100004.

15. Brown K, Sugrue M, Caldwell E, D`Amours S, Jalaudin B. South Western Sydney Regional Trauma Registry 10Year Report 1995-2004. 2006. p. 11-4.

16. Moore L, Clark DE. The value of trauma registries. Injury 2008;39:686-95.

17. Haider A, Saleem T, Leow J, Villegas C, Kisat M, Schneider E, et al. Influence of the National Trauma Data Bank on the study of trauma outcomes: is it time to set research best practices to further enhance its impact? J Am Coll Surg. 2012;214:756-68.

18. Mock C, Juillard C, Brundage S, Goosen J, Joshipura M. WHO Guidelines for trauma quality improvement pro- grammes Geneva: World Health Organisation 2009. 